CONFERENCE SUMMARIES 


\title{
THE EXTRAGALACTIC BACKGROUND RADIATION — 1989
}

\author{
Malcolm S. Longair \\ Royal Observatory \\ Blackford Hill \\ Edinburgh EH9 $3 H J, U K$
}

\section{NOSTALGIA}

This symposium has brought back many happy memories of the time 20 years ago when Rashid Sunyaev and I surveyed what was known about the extragalactic background radiation at that time. In one of our papers of 1969, we published a simple representation of the extragalactic background radiation spectrum, as it was known then (Figure 1). Extragalactic background radiation had been discovered in the radio, microwave, $\mathrm{X}$ - and $\boldsymbol{\gamma}$-ray wavebands but only upper limits were available in the intervening wavebands which have been the principal concern of this symposium. It is remarkable that, over the last 20 years, although the observational techniques have developed dramatically and there have been enormous astrophysical advances, the wavebands between $1 \mathrm{~mm}$ and $1 \mathrm{~nm}$ have continued to be very difficult indeed for studies of the extragalactic background.

In this brief summary, which is necessarily incomplete and personal, I will do no more than make some simple observations about studies of the extragalactic background radiation and its role in cosmological studies, then summarize the new results we have heard about this week and determine whether or not there are glaring inconsistencies between them.

\section{CAUTIONARY REMARKS}

\subsection{What Can One Really Hope to Learn?}

Let us consider first the case in which the background radiation is the integrated emission of discrete sources. All the textbooks describe how, in principle, the integrated extragalactic background radiation can be used as a discriminator of cosmological models and, if the Universe were constructed in a helpful way, this would indeed be possible. It is important, however, to recall what "helpful" means in this context. In the classical sum, which is repeated in Section 2.2, the background radiation associated with a population of sources of standard luminosity and spectrum is evaluated, assuming that the Universe is filled uniformly with them. In fact, the background radiation is the integrated emission of sources of widely differing luminosities and spectral types, there is convincing evidence for changes in the properties of different classes of source with cosmic epoch, and we cannot guarantee that we know all the principal contributors to the background. My own preference is therefore to regard the background radiation as a constraint upon world models and the properties of the sources which might make up the background rather than as a means of determining the geometry and dynamics of world models. 


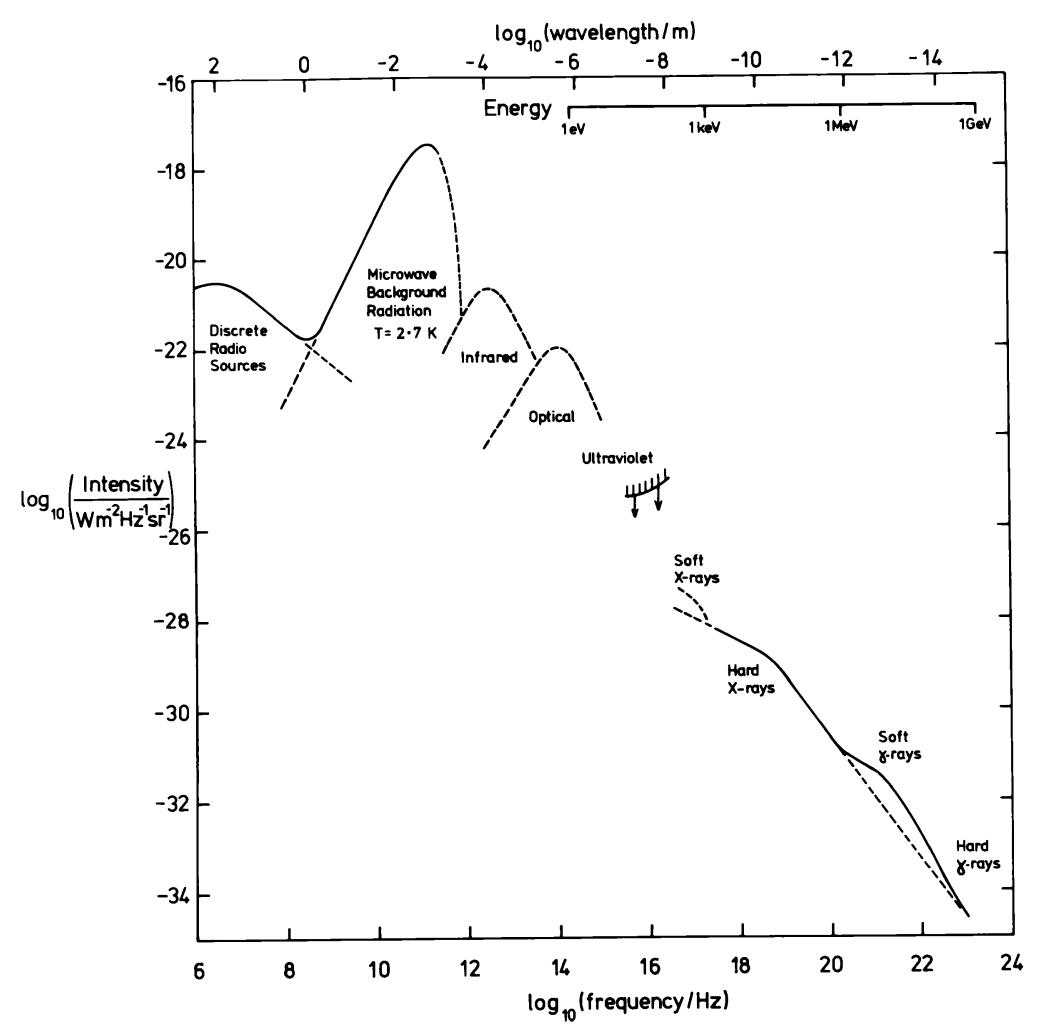

Figure 1. The spectrum of the extragalactic background radiation as it was known in 1969 (Longair and Sunyaev 1971). The solid lines indicate regions of the electromagnetic spectrum in which the extragalactic background had been measured. The dashed lines are theoretical estimates of the background radiation due to galaxies.

The case is somewhat different if the diffuse background radiation is not associated with discrete objects such as galaxies but rather with the matter between them. In these cases, the radiation content of the Universe is very much more closely related to physical processes that determine the present state of the matter and radiation content of the Universe and a quite different set of astrophysical tools is needed. The prime examples are the Microwave Background Radiation, which is convincingly associated with the cooled remnant of the hot quasiequilibrium phases of the Hot Big-Bang and the possibility that diffuse UV and X-radiation might be associated with the reheating of the intergalactic gas, either by photoionizing radiation from early generations of galaxies or quasars or by hot gas. In some cases there is a definite spectral signature associated with these processes. Part of the reason for our confidence in the basic correctness of the Hot Big-Bang picture is that the spectrum of the MBR matches very closely the predictions of the simple Big-Bang model. It would be very important if similar results could be found in relation to the processes associated with the subsequent reheating of the intergalactic gas.

Thus, if one is very lucky, studies of the background radiation can produce definitive information about the evolution of the Universe. If one is less lucky, there is a nasty mess to be disentangled and I believe one should be realistic about how much one can really learn in 
the more complex areas.

\subsection{The Olbers's Sum}

Let us begin with the classical calculation of the extragalactic light of the night sky for the uniform models of general relativity. I call this the Olbers's sum, although we now know the tortured history of the interpretation of the simple statement that "the sky is dark at night" (Harrison 1987). We carry out the calculation for a population of objects, each of which has luminosity $L(v)$ and number density at the present epoch $\rho_{0}(L)$. For illustration, I consider the intrinsic source spectra to be of power-law form $L(v) \propto v^{-\alpha}$. Finally, we have to consider the possibility that the properties of the sources evolve with cosmic epoch and we represent this by the evolution function $f(L, z, \ldots$.$) , which describes how the luminosity function of the sources$ changes with redshift as a function of luminosity $L$ and any other properties of the sources. In the case $f(L, z, \ldots)=$.1 , it is assumed that source population is uniform and that there is no change in the properties of the population with cosmic epoch. In my preferred notation, the background intensity is then

$$
I\left(v_{0}\right)=\int_{0}^{\infty} \frac{L\left(v_{0}\right)}{4 \pi D^{2}(1+z)^{1+\alpha}} f(L, z, \cdots) \rho_{0}(L) d V,
$$

where $d V$ is the element of comoving coordinate distance which, in my notation, is $d V=D^{2} d r$ for the volume element contained within one steradian and in the increment of comoving coordinate distance $d r$ at redshift $z$. In the notation which I use here, the increment of comoving coordinate distance is

$$
d r=\frac{c d z}{H_{0}(\Omega z+1)^{1 / 2}(1+z)}
$$

and the distance measure $D$ is given by

$$
D=\frac{2 c}{H_{0} \Omega^{2}(1+z)}\left[\Omega z+(\Omega-2)\left\{(\Omega z+1)^{1 / 2}-1\right\}\right] .
$$

These results are derived in the standard textbooks (see, e.g., Longair 1984). It will be noted that the $D$ 's cancel out in the numerator and denominator of expression (1) for the intensity of the background radiation. The result is that the intensity is given by the following expression:

$$
I\left(v_{0}\right)=\frac{L\left(v_{0}\right) \rho_{0}(L)}{4 \pi} \frac{c}{H_{0}} \int_{0}^{z} \frac{f(L, z, \cdots) d z}{(\Omega z+1)^{1 / 2}(1+z)^{2+\alpha}}
$$

In this integral, I have extended the integration only to redshift $z$ rather than infinity but obviously we obtain the total background by letting the upper limit go to infinity.

The integral (4) is the classical solution of Olbers's (or Halley's, or de Cheseaux's, or Kelvin's or ...) paradox. We obtain the classical Olbers's problem if we throw away all the redshift factors in the integral (4) and simply write $x=c z / H_{0}$ where $x$ is a standard Euclidean distance unlike $r$. Then,

$$
I\left(v_{0}\right)=\frac{L\left(v_{0}\right) \rho_{0}(L)}{4 \pi} \int_{0}^{x} d x .
$$

The classical Olbers's problem is the divergence of this integral as $x \rightarrow \infty$. The solution of the paradox is given by the integral (4) and it can be interpreted in various ways according to the 
taste of the reader. Formally, the most important factors are (i) the fact that at large redshifts $d r$ no longer increases linearly with redshift $z$ but in the limit of large redshifts varies as $d r \propto z^{-3 / 2} d z$ and (ii) the redshift factors $(1+z)^{-(1+\alpha)}$ in the expression for the flux density. Notice that the second factor includes the shape of the spectrum of the source. The net result of this calculation is that large redshift volume elements contribute less to the background than in the Euclidean calculation. The other point to note is that, when the integral over redshift is taken from 0 to $\infty$, inspection of the relation (3) shows that $D$ does not tend to infinity but to a finite value $D=2 c / H_{0} \Omega$. This may be thought of as corresponding to the fact that the Universe has a finite age (and extent) according to the classical models.

The important point to note is the dramatic effect the expansion of the Universe has upon the origin of the background radiation. It is interesting to ask out to what redshift we obtain half of the background radiation. Let us consider the simple case of the critical world model, $\Omega=1$ for sources with power law spectra with $\alpha=1$ and with no evolution $f(L, z, \cdots)=1$. Then, the integrated background radiation out to redshift $z$ is just proportional to $\left[1-(1+z)^{-5 / 2}\right]$. This means that in this model, half of the background radiation originates at redshifts less than 0.31 . The corresponding calculation for the $\Omega=0$ model gives $z(I(1 / 2))=0.42$. The combined effects of redshift attenuate very strongly the contributions to the background from larger redshifts.

This is an important point because it means that, unless there is strong evolution of the source populations with cosmic epoch, we do not expect the background due to discrete sources to originate from remotely large redshifts but from relatively modest redshifts. It also means that, in reasonably uniform world models, it should not be very difficult to identify the main discrete source contributors to the background radiation. Notice that the principal reason for this behavior is the decrease in the comoving volume elements with increasing redshift. Whereas at small redshifts, $z \ll 1$, the volume elements increase with redshift as $z^{2} d z$, at redshifts $\Omega z \gg 1$, the volume elements decrease with redshift as $d z / z^{3 / 2}$.

If the background from discrete sources is to come from large redshifts, then there must be very strong changes in the comoving space density of sources with cosmic epoch - the combined effect of the decrease in the volume elements with increasing redshift and the effects of redshift upon the intensities of the sources must be overcome. A good example of this, in fact the only example we know of, is provided by the radio background emission. As discussed by Wall in his review of the radio background radiation, the observed isotropic component of the radio background radiation at meter wavelengths corresponds to a brightness temperature $T_{b}=\left(\lambda^{2} / 2 k\right) I_{v}=20-30 \mathrm{~K}$. This brightness temperature can be compared with the values expected from the observed local space density of radio sources and with the observed source counts. If there were no evolution of the radio source population, we would expect about $4 \mathrm{~K}$ from normal galaxies and about $1 \mathrm{~K}$ from the strong radio galaxies and quasars. In fact, because of their very strong evolution with cosmic epoch, the contribution of the strong radio galaxies and quasars amounts to about $16-19 \mathrm{~K}$ so that the total background emission at meter wavelengths can probably be accounted for in terms of the integrated emission of discrete sources. Notice that the information about evolution comes from the anomalies in the radio source counts and the spatial distribution of the radio sources. The radio background simply assures us that it is unlikely that there is any other major contributor to the radio background emission.

The reason for this big difference between the expected background from a uniform distribution of sources and the observed values is entirely due to the effects of strong evolution of the luminosity function of the radio sources with cosmic epoch. The evolution can be described by simple changes of the radio luminosity function with redshift or cosmic epoch 
and Figure 2 shows a recent successful model due to Dunlop and Peacock (1989). In terms of the change of the comoving space density of sources, it can be seen that, at a redshift of about 2, the powerful radio sources with $P_{2.7} \gg 10^{26} \mathrm{~W} \mathrm{~m}^{-2} \mathrm{~Hz}^{-1}$ are about 1000 times more common than they are at the present epoch. This example gives some idea of the amount of evolution necessary to overcome the combined effects of redshift and the decrease in comoving volume at large redshifts.

\section{Steep-Spectrum}

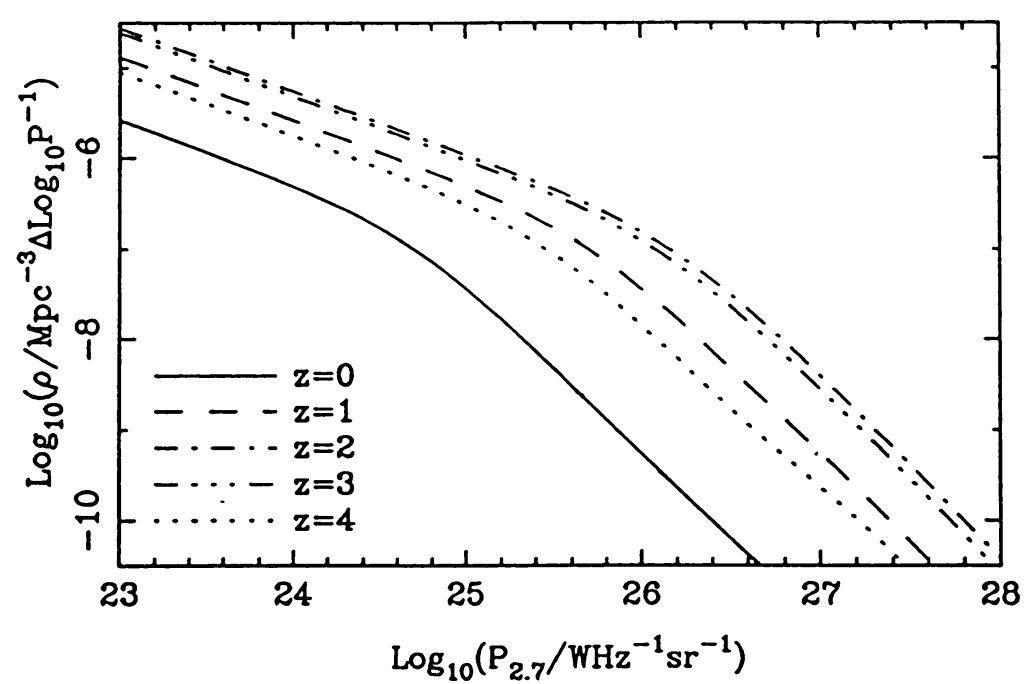

Figure 2. Illustrating the changes in the form of the radio luminosity function per unit comoving volume with redshift. This model can account for all the present observations and is described in terms of luminosity evolution in which the luminosity function of strong radio sources is shifted to higher radio luminosities at early epochs. The maximum luminosity occurs at redshifts $z-2-3$. At larger redshifts, the average luminosity decreases from the maximum value (Dunlop and Peacock 1989).

It is interesting that the determination of the extragalactic background emission at meter wavelengths has not received much attention since the 1960's, except for those experiments designed to extract the component of the MBR still present at these wavelengths.

\subsection{Counts of Sources and the Background Radiation}

It is useful to compare the information provided by the counts of sources and by the background radiation associated with them. The standard derivation of the famous $3 / 2$ power law follows from considering the numbers of sources of luminosity $L$ which could contribute to the background radiation. If $\rho_{0}(L)$ is the local space density of sources of luminosity $L$, these can be observed to a distance $x=(L / 4 \pi S)^{1 / 2}$ at a limiting flux density $S$. In a Euclidean Universe, the number of sources per steradian counted out to this distance must be $1 / 3 x^{3} \rho_{0}(L)$, i.e. the number of sources with flux densities greater than $S$ is

$$
N(\geq S) \propto\left[\rho_{0}(L) L^{3 / 2}\right] S^{-3 / 2} .
$$


On the other hand, as shown by the integral (4), the background radiation is proportional to a different combination of $L$ and $\rho$

$$
I(v) \propto\left[\rho_{0}(L) L\right] .
$$

Now suppose that a background of intensity $I$ is observed and it is to be attributed to discrete sources. Then, the number of sources which make up the background is found by eliminating $\rho_{0}(L)$ between the expressions (6) and (7):

$$
N(\geq S) \propto L^{1 / 2} S^{-3 / 2} \text {. }
$$

This expression makes the simple point that, if one cannot identify the sources responsible for the background at a particular flux density, then they must be of lower intrinsic luminosity than the other sources in the sample and have correspondingly greater space density to produce the observed background intensity. This argument is of interest in connection with the origin of the X-ray background which remains a matter of considerable debate. As described by Setti, known types of X-ray emitter, in particular the Seyfert galaxies, can make up a significant fraction of the background radiation at X-ray energies of about $1 \mathrm{keV}$. It is not yet established if these are really the source of the background because it is not known if they have the correct $\mathrm{X}$-ray spectrum, in particular whether or not they possess a turnover in their spectra at about 20-30 keV. If they are not, the sources of the background would have to be sought among a population of lower luminosity but more common types of source. Indeed, this is a basic problem about accounting for all the background radiation. It is not at all difficult to invent low luminosity classes of source which could make a significant contribution to the background radiation.

Let us look at the counts themselves. The background radiation from a population of sources with differential source count $d N \propto S^{-\beta} d S$ results in a background intensity:

$$
I \propto \int S d N \propto \int S^{-(\beta-1)} d S \propto S_{\min }^{-(\beta-2)} .
$$

Now, for a Euclidean population of sources $\beta=2.5$. In real world models, the slope is 2.5 at small redshifts but decreases because of the effects of redshift at $z \geq 0.2$. There is a critical slope corresponding to $\beta=2$ at which the maximum contribution to the background is obtained. This result is indeed found to be the case for the radio background emission - most of the background is associated with the excess of sources at high flux densities.

Another interesting example is the case of the optical counts of galaxies presented by Tyson. According to his results, the differential counts of galaxies flatten at the very faintest apparent magnitudes. This means that, unless there is an increase in the counts, again due to a new population of very faint galaxies, we have probably seen most of the background light due to faint galaxies.

Another general feature of discrete source models for the background is that there is inevitably a smearing in redshift of the spectrum of the main contributors to the background. In general, the background originates from a redshift range $\Delta z / z \sim 1$ and there is therefore a corresponding smearing of $\Delta v / v$ in the observed spectrum of the background radiation. Thus, any sharp emitted feature is spread out into a broad feature by the cosmological redshift. This may prove to be a problem for discrete source models of the excess radiation observed in the submillimeter and infrared wavebands by Matsumoto and his colleagues.

Another topic of considerable interest is the origin of fluctuations in the background radiation due to discrete sources. This is a well-known solved problem, the simplest presentation that I know of being that of Scheuer (1974), who first solved this problem to deal with confusion in radio source surveys. The simple result of these studies is that the fluctuations in 
intensity provide information about a surface density of sources of roughly one per beamwidth with flux densities corresponding roughly to $S \sim \Delta I$. This result would only be seriously in error if the distribution of sources were dramatically nonuniform; for example, if all the sources had the same intrinsic luminosity at a single redshift. Thus, so far as discrete source models are concerned, the fluctuations should be considered as a means of obtaining further information about the source counts at flux density levels less than those at which individual sources can be clearly distinguished.

The other approach to the interpretation of fluctuations in the background radiation is to look for a signal in the correlation function of the fluctuations. This has been carried out succesfully in the optical waveband by Shectman (1974) who found a clear signature corresponding to the two point correlation function for galaxies. As Peebles described in his presentation, the observed fluctuation spectrum is in quite remarkable agreement with the standard correlation function found in studies of large samples of galaxies. The most recent application of this approach is the heroic work of Martin and Bowyer (1989) reported by Bowyer. From their short rocket flight, they were able to make a survey of a small region of sky and found a significant correlated signal among the spatial distribution of counts. With a number of reasonable assumptions, they were able to show that they have detected the ultraviolet emission from galaxies. We will return to the significance of their results in Section 3.

Similar analyses have been carried out for the fluctuations in the X-ray background as observed by the HEAO 1 A-2 experiment (Persic et al., 1989). The binning of the background counts was in $3^{\circ}$ pixels. No significant signal was found in the two-dimensional autocorrelation function on all angular scales greater than $3^{\circ}$. This constrains the clustering scale of the sources which might make up the background radiation to scales less than about $50 \mathrm{Mpc}$. They show that the observed upper limits to the clustering would be consistent with the observed cross-correlation function for galaxies and clusters of galaxies. A similar analysis has been carried out by Barcons and Fabian (1989) who have studied fluctations in five deep Einstein IPC fields. A signal is observed on the scale of $5^{\prime}$ but it is not certain that this is of astrophysical origin. A maximum comoving clustering scale of $10 h^{-1} \mathrm{Mpc}$ is found. This rules out models in which the background is associated with clusters of galaxies at low redshifts, $z \leq 1$.

\subsection{The Diffuse Extragalactic Background Emission}

The predicted diffuse emission from the intergalactic medium is strongly dependent upon the physical processes responsible for heating and cooling the matter and radiation. The best example is the MBR which has a spectrum which closely reflects the quasi-equilibrium conditions which pertained when the matter and radiation were strongly coupled in the early Universe. The spectrum of the MBR is Planckian because it has an enormous heat capacity and distorting it from a blackbody spectrum requires quite enormous inputs of energy. The beauty of the Hot Big-Bang model is that the MBR comes very naturally out of the requirements that the temperature of the matter be hot enough for nuclear reactions to take place and that, in such a high temperature photon-dominated plasma, there is time for the nuclear interactions to take place. This aspect of the Hot Big Bang was described by Harwit in his thoughtprovoking introduction to this symposium (Harwit 1989, this volume). The argument is as close as one is likely to get to a purely thermodynamic argument in astrophysical cosmology.

The situation is quite different in the case of the diffuse radiation which might be associated with the reheating of the intergalactic plasma. There remains the enigma of the hard $X$ ray background - it can be accounted for remarkably successfully by a hot thermal 
bremsstrahlung spectrum with electron temperature corresponding to $k T \approx 30 \mathrm{keV}$. If this gas were uniformly distributed thoughout the Universe, the mass of intergalactic gas would correspond roughly to the critical density and the energy requirements to heat such a gas to $3 \times 10^{8} \mathrm{~K}$ are quite enormous. This hypothesis would also be in conflict with the limits to the mean baryon density of the Universe which come from the arguments concerning primordial nucleosynthesis $\Omega \leq 0.05 h^{-2}$. The solution is to clump the gas because the emission per unit volume increases as $N_{e}^{2}$. However, there is no consensus about this model and, even if the matter is clumped, there remains the problem of ionizing all the matter between the clumps.

The advantage of the photoionization model for the ionization of the intergalactic gas is that it has much less severe energy demands and galaxies and quasars can provide powerful sources of ionizing radiation. In this case, the intergalactic gas does not have to be heated to a very high temperature. Predictions have been made of the Lyman continuum flux needed to photoionize the intergalactic gas but the origin of this hypothetical flux of radiation is not known and its spectrum is a matter of speculation.

Just as in the case of discrete source models, fluctuations in the diffuse background intensity can provide clues to its origin. The potential of fluctuations in the MBR for providing information about a very wide variety of astrophysical problems has been the subject of most study. The reasons for this are not hard to discern. First of all, the background radiation is convincingly associated with the adiabatically cooled remnant of the Hot Big-Bang. We can therefore think of the MBR as originating from a "photosphere" at about the epoch of recombination. The variation of the degree of ionization through this photosphere has been worked out in detail by Jones and Wyse (1985) who show how rapidly the optical depth to Thomson scattering increases with redshift through the epoch of recombination. In fact, with increasing redshift, the optical depth of the intergalactic gas to Thomson scattering becomes unity at a redshift of 1070. An approximate expression for the optical depth to Thomson scattering through the neighboring redshift range is given by the expression

$$
\tau=0.37\left[\frac{z}{1000}\right)^{14.25} \text {. }
$$

We can now work out the range of redshifts at which the photons of the MBR were last scattered. The probability distribution of last scattering is given by the simple function

$$
\frac{d p}{d z}=e^{-\tau} d \frac{\tau}{d z}
$$

This function is rather narrow in redshift, a good approximation being a Gaussian distribution with standard deviation 80 in redshift. It is through this rather narrow range in redshift that primordial density fluctuations impart their fingerprints to the MBR. It is interesting that an interval of 80 in redshift at the epoch of recombination corresponds to a physical size of $7\left(\Omega h^{2}\right)^{-1 / 2} \mathrm{Mpc}$ at the present epoch. It is not a particularly straightforward (or intuitive) business to relate the fluctuations in the density of the matter (normal or cold) to the temperature fluctuations, particularly if the spectrum of the fluctuations is given in terms of the primordial spectrum in k-space. The point is that, although there is a one-to-one relation between the angular scale of fluctuations and the physical scale at the decoupling epoch, waves of a wide range of wave-numbers can contribute significantly to the fluctuations expected on a given physical scale. The net result is that the expected amplitude of the temperature fluctuations in the MBR depends upon the chosen spectrum of initial fluctuations and how they are supposed to evolve with cosmic epoch. We have to read very carefully the input assumptions in any of the calculations. 
In addition to these problems, we have to worry about other contributions to the fluctuation spectrum - the reheating of the intergalactic gas which is expected to be a non-uniform process, the Sachs-Wolfe effect on large physical scales, the Sunyaev-Zeldovich effect due to regions of hot gas in the interglactic plasma, the effects of discrete sources on the background fluctuations. I confess that I worry about how unambiguous the interpretation of the fluctuations will be when they are observed with good signal to noise ratio. While I am wholly convinced of the importance of making a major effort to define the spectrum of fluctuations in the Microwave Background Radiation, I am convinced that it needs a really major increase in sensitivity before it will realize its enormous astrophysical potential.

\section{THE EXTRAGALACTIC BACKGROUND RADIATION}

The most useful thing I can do is to try to put all the observations we have heard about this week into context. I have summarized many of the data in Figure 3 in which I have converted all the reported measurements and limits onto a uniform scale of $v I_{v}=\lambda I_{\lambda}$ in SI units. $I_{v}$ (or $I_{\lambda}$ ) is the intensity of radiation in units of power per unit frequency range (or per unit wavelength) per steradian and so $v I_{v}$ is a measure of the energy emitted at frequency $v$. This means that the total area under a plot of $v I_{v}$ against frequency gives the energy emitted in that frequency window. The SI units of $v I_{v}$ are $\mathrm{W} \mathrm{m}^{-2} \mathrm{sr}^{-1}$; for reference, the axes of Figure 3 also show the values of $v I_{v}$ in erg $\mathrm{cm}^{-2} \mathrm{~s}^{-1} \mathrm{sr}^{-1}$. Can I strongly recommend that standardization on SI units would be of great benefit to all astronomers, particularly when cross-waveband studies are undertaken.

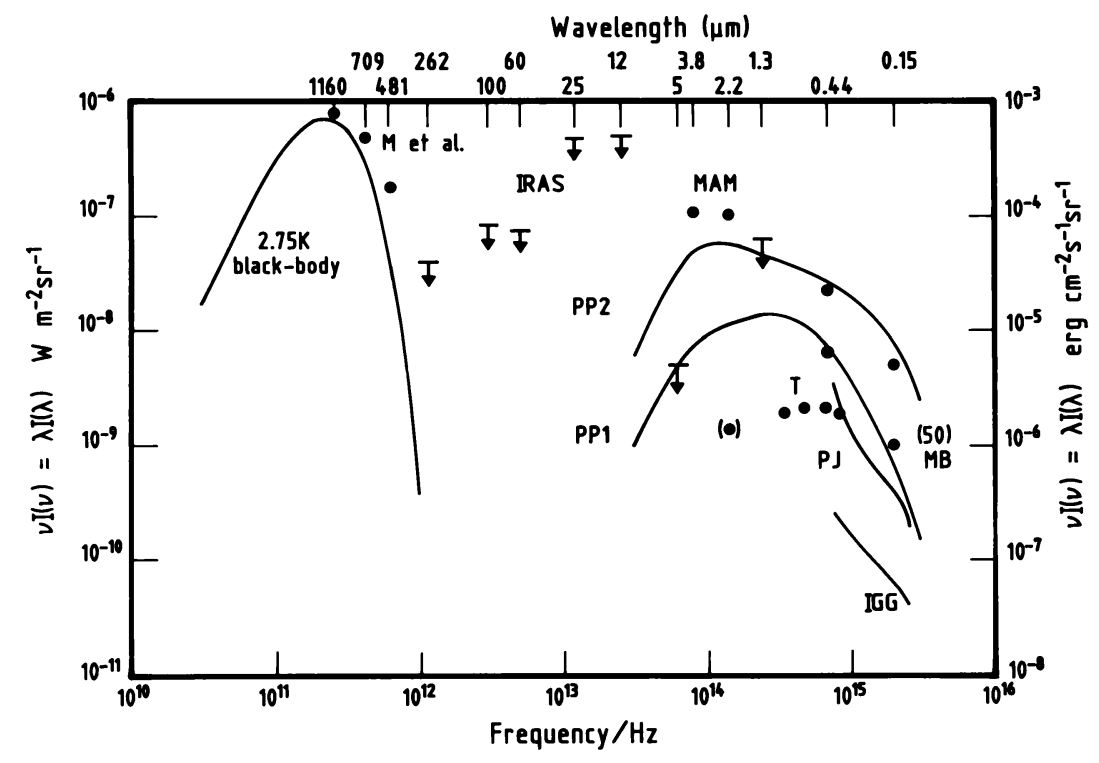

Figure 3. The spectrum of the extragalactic background radiation. Details of the measurements and upper limits are given in the papers included in this volume. 
Let me go through the values I have adopted in compiling Figure 3. At centimeter, millimeter and submillimeter wavelengths, I have shown a blackbody curve of radiation temperature $2.75 \mathrm{~K}$. The submillimeter measurements of Matsumoto et al. (1988) at 1.16, 0.709, and $0.481 \mathrm{~mm}$ and the upper limit at $0.262 \mathrm{~mm}$ are shown. Boulanger kindly provided me with upper limits to the extragalactic background radiation from the IRAS survey at wavelengths of $100,60,25$, and $12 \mu \mathrm{m}$. In the $1-5 \mu \mathrm{m}$ waveband, I show the measurements of Matsumoto, Akiba, and Murakami (1988) at 2.2 and $3.8 \mu \mathrm{m}$ as well as their upper limits at 1.3 and $5 \mu \mathrm{m}$. Matsumoto noted that there may be some contamination from the rocket exhaust in the $3 \mu \mathrm{m}$ observations and so it should probably be treated as an upper limit.

In the optical waveband, I have shown two measurements at $0.440 \mu \mathrm{m}$. The higher intensity is due to Mattila and Schnur and corresponds to his intensity measurement of $(6.5 \pm 2.5) \times 10^{-12} \mathrm{~W} \mathrm{~m}^{-2} \mathrm{sr}^{-1} \AA^{-1}$. The lower figure corresponds to Toller's measurement of (1.3 \pm 1.3$) S_{10}$ units from the Pioneer-10 experiment which measured the optical background radiation when the spacecraft was at a distance of $3 \mathrm{AU}$ from the Sun. At this distance, the background from zodaical light should be small. Mattila interprets these results as being consistent in that the $2 \sigma$ upper limit to Toller's measurement corresponds to $\lambda I_{\lambda} \leq 4.5 \times 10^{-12} \mathrm{~W}$ $\mathrm{m}^{-2} \mathrm{sr}^{-1} \AA^{-1}$. Finally, in the ultraviolet waveband, I have plotted the value of $v I_{v}$ corresponding to 250 photon units which is a reasonable compromise from the presentations of Bowyer, Lequeux, Henry, Paresce and Onaka of the residual ultraviolet background.

I have superimposed upon these measurements other estimates of the background due to discrete objects. The four points marked $T$ are the background radiations due to galaxies in the optical waveband from Tyson's counts of galaxies to about 30th magnitude. The point shown in brackets at $2.2 \mu \mathrm{m}$ is a prediction by Tyson of the corresponding background intensity in the infrared waveband. Notice that this is simply the integral of the corrected counts of galaxies and is not an extrapolation to infinite magnitude. The point marked $\mathrm{MB}(50)$ is Martin and Bowyer's estimate of the background due to galaxies in the ultraviolet waveband on the basis of the cross-correlation function derived from the photon counts in their brief rocket survey.

Finally, I have transcribed various theoretical models of the extragalactic background onto Figure 3. The models PP1 and PP2 are the old models of the optical and infrared backgrounds due to galaxies evaluated by Partridge and Peebles (1967) and span the range of scenarios discussed by them. The line marked PJ is the model described by Paresce and Jakobsen (1980) for the ultraviolet background due to galaxies. The locus marked IGG is the ultraviolet background radiation needed to photoionize the intergalactic gas, also presented by Paresce and Jakobsen.

My interpretation of all these data is that there is no major discrepancy between all the observations, the limits and the theoretical estimates. Let us look at the individual wavebands in turn.

\subsection{The Submillimeter Background Radiation}

De Zotti and Partridge discussed the problem of accounting for the excess submillimeter background by various models. De Zotti's analysis suggested that Compton scattering of photons of the Microwave Background Radiation provides a poor fit to the observed excess. The question hangs upon the accuracy of the measurements of the background radiation temperature at millimeter wavelengths and of the submillimeter observations from the Berkeley-Nagoya balloon experiment. The discrepancy results from literal interpretation of errors on the observations quoted by the observers. I believe it is often dangerous to give excessive weight to this type of argument. The example I like to quote is the succession measurements of the velocity 
of light between 1945 and 1960 . It is remarkable that, in nearly every case, each successive improved measurement was outside the formal errors of the previous measurement and the present adopted value is outside the experimental errors of many of the best experiments (Longair 1989). Notice that these are the results of laboratory experiments and not of astronomical observations. This argument simply emphasizes again how important high precision measurements of the MBR are. I personally would be somewhat reluctant to exclude the Compton model at this stage. A value of the Compton scattering parameter $y-0.02$ would be required. De Zotti made the interesting point that, if the X-ray background were due to the bremsstrahlung of hot intergalactic gas, greater distortions of the MBR spectrum than are observed would be expected.

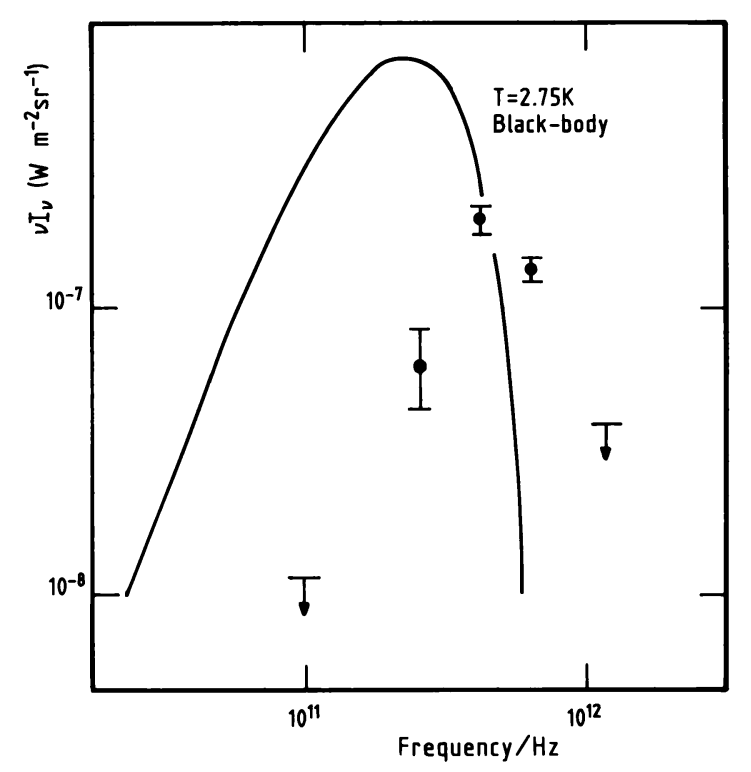

Figure 4. The energy densities in the MBR assumed to have a blackbody spectrum at $2.75 \mathrm{~K}$ and in the excess submillimeter radiation reported by Matsumoto et al. (1989).

The alternative is that there must be some additional component present at submillimeter wavelengths. In Figure 4, I show what happens if one subtracts from the observations of Matsumoto et al. a perfect blackbody spectrum with radiation temperature $2.75 \mathrm{~K}$. I have assumed no error in the blackbody spectrum so that the errors correspond to the errors on the total temperature quoted by Matsumoto et al. I have included an upper limit at $3 \mathrm{~mm}$ $\left(10^{11} \mathrm{~Hz}\right)$ corresponding to a maximum temperature contribution of $0.1 \mathrm{~K}$ to the excess. Figure 4 shows the relative contributions of the MBR and the submillimeter excess to the total intensity. By comparing the areas under the curves, the figure makes the point that the total energy in the submillimeter background corresponds to about $10 \%$ of that in the $2.75 \mathrm{~K}$ blackbody. This means that it is a very large amount of energy, corresponding to a density parameter in submillimeter radiation of $\Omega_{\text {rad }}-3 \times 10^{-6} h^{-2}$. In addition, Figure 4 makes the point very clearly that the excess radiation must have a very peaked spectrum. Comparison of the spectra of the $2.75 \mathrm{~K}$ blackbody and the excess shows that a spectrum shaped similarly to that of a 
blackbody with temperature $T \sim 6-7 \mathrm{~K}$ would just provide a reasonable fit to the excess. The very peaked spectrum of the excess provides a severe constraint upon possible models of its origin. Any smearing of the Planck spectrum would cause problems, either because of the effect of redshift or of any dispersion in the properties of the sources of the radiation.

One possibility discussed by a number of authors is that the excess is due to dust associated with Population III stars in primaeval galaxies. If the radiation were due to dust, the intrinsic emission spectrum would be narrower than that of a blackbody spectrum because the dust would be optically thin at long wavelengths and its emissivity $\kappa \alpha v^{2}$. As a result, the long-wavelength spectrum can be approximated by a spectrum of the form $v I^{v} \alpha v^{5}$ with an exponential cut off at short wavelengths. This spectrum can be fitted satisfactorily to the excess seen in Figure 4 and Matsumoto et al. used such a model with dust grains at a temperature $T=3.55(1+z) \mathrm{K}$ as a possible model for the background radiation. Again, however, one should worry about the processes which could lead to broadening of the emission spectrum. There would have to be essentially no dispersion in the redshift from which the energy originated and no dispersion in the intrinsic temperature distribution of the dust grains. One should also worry about how realistic these constraints are. As Boulanger pointed out, the spectrum of the submillimeter excess is significantly narrower than even the most peaked IRAS galaxy energy spectrum in the far-infrared waveband.

There remains, of course, the question of the reality of the excess. The beautiful Berkeley-Nagoya experiment is operating in a very difficult region of the electromagnetic spectrum, in which the spectrum of the MBR is decreasing exponentially. It would not require too large uncertainties to significantly change conclusions about the nature of the excess. The more detailed observations to be made by COBE will be crucial in elucidating these key questions for cosmology.

\subsection{The Far Infrared Background}

Perhaps not surprisingly, the upper limits to the intensity of the far infrared background, 12 to $100 \mu \mathrm{m}$, have not changed a great deal since 1970 , largely because the brightness of the extragalactic sky is masked by zodaical light and by high latitude cirrus. Nonetheless, the limits shown in Figure 3 are useful in setting limits to the amount of energy which could be reradiated in the infrared waveband by young galaxies. From Figure 3, it can be seen that already less than about ten times the optical luminosity of galaxies could be liberated in the far infrared background. This enables us to set some limits to the early evolution of galaxies. As pointed out by Matsumoto, it is not clear how much better one can do because the high latitude background radiation in the 12 to $100 \mu \mathrm{m}$ waveband is dominated by the emission of cirrus and zodiacal light.

\subsection{The 1 - $5 \mu \mathrm{m}$ Background}

The situation here resembles that in the submillimeter waveband. Matsumoto, Akiba and Murakami claim to have detected a background component in the $2.2 \mu \mathrm{m}$ waveband. Inspection of Figure 3 shows that, if this observation is correct, the spectrum of the radiation must be rather peaked at about $2 \mu \mathrm{m}$ because of the strong upper limits at 1.3 and $5 \mu \mathrm{m}$ and the warning by the observers that it may be safer to treat the observation at $3.8 \mu \mathrm{m}$ as an upper limit. A number of points can be made about this result.

1. This intensity exceeds Tyson's prediction of the background radiation at $2 \mu \mathrm{m}$ by about a factor of 50. It is not outside the conceivable ranges of intensities of the 
background radiation due to strongly evolving young galaxies (see the model PP2 in Figure 3) but the spectral signature is all wrong.

2. Matsumoto mentioned the possibility that the excess may be due to strongly redshifted Lyman- $\alpha$ from the early phases of galaxy formation. In this case, one finds immediately the redshift at which most of the star formation activity must be taking place, namely a redshift $(1+z)=2.2 / 0.122$ i.e., $z \sim 17$. In principle, this cannot be excluded but it is certainly a larger redshift than the values inferred by Tyson from the mean color of the background due to faint galaxies.

This remains a puzzle; it will be necessary to obtain independent confirmation of the reality of this feature.

\subsection{The Optical Background Radiation}

The optical background radiation remains as tantalizing as ever. The range of current possibilities is illustrated in Figure 3. Many workers believe that the observed value of the extragalactic background cannot be much more than about $1 S_{10}$ unit corresponding to an intensity of about $5 \times 10^{-9} \mathrm{~W} \mathrm{~m}^{-2} \mathrm{sr}^{-1}$ at $440 \mathrm{~nm}$. The one significant exception to this is the observation of Mattila and Schnur who report a measurement corresponding to $(2.3 \pm 0.8) \times 10^{-8}$ $\mathrm{W} \mathrm{m} \mathrm{m}^{-2} \mathrm{sr}^{-1}$. According to Mattila, these results are consistent within the uncertainties of both measurements. Mattila and Schnur use the dark cloud method in which the intensity of the background in the direction of a dark cloud $I_{\text {dark }}$ at high galactic latitude is compared with the intensity in a neighboring direction away from the dark cloud $I_{t o t}$. Naively one might imagine that the background originating from beyond the cloud would be $I_{t o t}-I_{\text {dark }}$. The problem with the naive argument is that the background in the direction of the dark cloud exceeds that in the unobscured direction because of scattering of the optical emission of our own Galaxy by the dust in the dark cloud! The net result is that the proper interpretation of the results of the dark cloud method depends upon the scattering properties of the grains and this is a subject of debate. I refer readers to Mattila's paper in which the major advances made in using this technique over the last decade are described and it is certainly impressive. My own concerns relate to the problem of how secure the background estimates are in view of the uncertainties in the properties of the dust grains. These problems are of considerable technical complexity and plainly require much further study.

The total background due to galaxies can be estimated from the various counts which now extend to quite remarkably faint magnitudes. It is very encouraging that, as described by Tyson and Shanks, the counts of faint galaxies made by independent workers are now in agreement down to about 25th magnitude. There is an excess of faint galaxies which is naturally interpreted as the effects of the evolution of their intrinsic luminosities and spectra. Tyson's counts extend to about 30th magnitude and he made the important statement that the differential counts of galaxies flatten off at the very faintest magnitudes so that there is roughly the same number of galaxies per magnitude interval. As discussed in Section 2.3, this means that, unless the galaxy counts begin to increase again at even fainter magnitudes, the bulk of the galactic light contribution to the background radiation has already been seen. The integrated intensity from Tyson's counts corresponds to about half or a third of the fiducial value of about $5 \times 10^{-9} \mathrm{~W} \mathrm{~m}^{-2} \mathrm{sr}^{-1}$. This does not leave much room for any other major contributor to the optical background radiation.

Tyson's analysis raises a host of interesting questions which will undoubtedly be addressed vigorously over the coming years. First of all, it will be very important to obtain 
independent counts to the very faintest magnitudes. I say this not because I have doubts about the analysis of Tyson and his colleagues but because the history of galaxy and source counting is fraught with complexities which take some time to resolve. Guhathakurta's paper is a good example of the type of problem which has to be taken seriously in these studies.

Second, Tyson provided intriguing information related to the redshift distribution of the galaxies in his sample. As can be seen from the points displayed in Figure 3, there is no evidence for any strong decrease at short wavelengths in the spectral distribution of the background radiation. This suggests that the Lyman discontinuity has not been redshifted into the accessible waveband and, on this basis, Tyson infers that the bulk of the galaxies counted probably lie in the redshift range 1 to 3 . This would be entirely consistent with the rules which I outlined in section 2.2 about the likely redshift range over which most of the background radiation should originate. This suggests that the distribution of galaxies must display some sort of cutoff at redshifts greater than about 3, a phenomenon similar to that found in the distribution of radio galaxies and quasars at large redshift. Is history repeating itself in the case of the counts of galaxies? If Tyson is right, we might already have seen most of the galaxies there are to be seen!

A third point concerns the blueness of the galaxies observed by Tyson at the faintest magnitudes. It is quite remarkable that the galaxies are so blue that their integrated intensity shows a maximum in the red region of the spectrum rather than in the $1-5 \mu \mathrm{m}$ waveband which would be expected because of the effects of redshift upon the standard spectrum of an elliptical or spiral galaxy. This effect is illustrated by the PP models shown in Figure 1. This is a very important result for astrophysical cosmology and needs much further study.

There is a clear need to compare the theoretical models of the evolution of galaxies with these results in much more detail. In recent years, many improved models of galactic evolution have become available which attempt to take into account all the recent evidence on the properties of galaxies in the optical, ultraviolet and infrared wavebands. The models of Partridge and Peebles, Tinsley and Bruzual should be superceded by more sophisticated models. I am most familiar with the work of my colleagues Guiderdoni and Rocca-Volmerange who have shown that constraints can be obtained on both cosmological parameters and the evolution of galaxies using the best data available. I would strongly recommend that these types of theoretical analysis be extended to the infrared waveband, both for the continuum emission due to stars which should be the main contributors in the 1 to $5 \mu \mathrm{m}$ waveband and for the dust emission which might be associated with early generations of star formation in galaxies at large redshifts which would make important contributions to the background in the far infrared waveband, 30 to $300 \mu \mathrm{m}$. The limits provided by the Japanese experiments at 5 and $262 \mu \mathrm{m}$ are of particular interest in this regard.

\subsection{The Ultraviolet Background Radiation}

A clear message came through from the various discussions of the existence or otherwise of the ultraviolet background radiation which was best summarized by Paresce. There is a correlation between the intensity of the ultraviolet background radiation and the column density of neutral hydrogen but, extrapolating zero column density, there is a residual component of about 200 to 300 photon units. In Figure $3 \mathrm{I}$ have plotted a value of $5 \times 10^{-9} \mathrm{~W} \mathrm{~m}^{-2} \mathrm{sr}^{-1}$, which corresponds to 250 photon units at $150 \mathrm{~nm}$. This flux is only an upper limit to the extragalactic flux since there are likely to be other contributions to the high latitude ultraviolet radiation field, among which I would highlight the radiation field necessary to produce the ionized gas observed at high latitudes in the remarkable observations by Reynolds as well as the likely 
importance of fluorescence by molecular hydrogen and two-photon emission.

The estimate of the extragalactic flux due to galaxies of 50 photon units by Martin and Bowyer, corresponding to $1 \mathrm{nW} \mathrm{m} \mathrm{mr}^{-1}$, is a remarkable achievement. The approach is similar to that of Shectman in the optical waveband and it establishes that a correlated signal is present among the photon counts recorded in the experiment which corresponds closely to the two-point correlation function for galaxies. Their estimate of the background is included in Figure 3 and it can be seen that no one would be at all surprised if this is indeed the correct answer. The old models of Partridge and Peebles and that adopted by Paresce and Jakobsen (1980) show that a flux similar to that observed would not be unexpected. The case for a dedicated survey mission for these wavebands is very convincing.

I have also included in the ultraviolet part of Figure 3 one of the models adopted by Paresce and Jakobsen for the ultraviolet background radiation needed to photoionize the intergalactic gas and it can be seen that it is within about an order of magntiude of being observable. Of course, how well it can be distinguished against the background due to galaxies is a problem. It should, however, be relatively stronger at shorter wavelengths and so there might be the possibility of observing it in the far UV waveband. One approach to the far ultraviolet waveband, which was not mentioned explicitly during the Symposium, was the technique of using the existence of neutral hydrogen clouds in intergalactic space to set limits to the far ultraviolet background intensity (Sunyaev 1969). In discussions, I had the impression that the photoioning flux density shown in Figure 3 would be consistent with observations of diffuse neutral hydrogen in Lyman- $\alpha$ absorption clouds and with the existence of diffuse intergalactic HI clouds at the present day. It would be worthwhile checking this.

\subsection{The $X$ - and $\gamma$-ray Background}

Finally, I mention briefly the $X$ and $\gamma$ ray background radiation. I must confess that $I$ find the origin of the X-ray background to be one of the most frustrating problems in cosmology. The $\mathrm{X}$-ray background was discovered in the very first rocket $\mathrm{X}$-ray observations of the sky but there is still no convincing explanation of its intensity and spectrum. Setti surveyed the many possibilities and the problems associated with each of them. At low energies, $h \mathrm{~V} \sim 1 \mathrm{keV}$, the results of the Einstein X-ray observatory can be used to make estimates of the contributions from various classes of discrete source and there is agreement that a significant fraction of the background could be due to active galaxies but exactly how much is not clear. The exact value depends upon the unknown evolutionary properties of the sources with cosmic epoch. For the Seyfert galaxies, which are expected to be the main contributors, we simply do not know the answer.

The frustrating part of the story is that the background has a clear spectral signature the well-known break in the continuum spectrum at about $30 \mathrm{keV}$ - and this should be mirrored in the spectra of the sources themselves. Unfortunately, very few sources have been observed in the difficult 10 to $100 \mathrm{keV}$ waveband. Those which have been observed, which include many of the brightest active galaxies in the sky such as 3C 273 and NGC 4151, do not show the necessary break in their spectra. Indeed, these data have been used in quite the opposite way by proponents of $\gamma$-ray astronomy. They use these data to show that active galaxies emit most of their energy at about 1-3 MeV which is exactly what the X-ray cosmologist does not want!

The result is that there remains plenty of speculation about possible sources of the X-ray background. It is still a remarkable fact that the X-ray background spectrum can be very nicely described by a thermal bremsstrahlung spectrum and I am sure that, even once 
allowance is made for the contribution of sources, this statement would remain correct. There are problems with attributing the background to diffuse hot gas at $k T \sim 30 \mathrm{keV}$, largely because of the enormous energy requirements. The solution is then to clump the gas so that less energy is required. How far one wishes to go is a matter of speculation. Among ideas described at this meeting have been the thermal emission from the hot gas clouds surrounding radio galaxies and other active galaxies and clumping the gas even further, high mass X-ray binaries in normal galaxies (Griffiths 1989, this volume). The clear requirement is for high sensitivity in the 10 to $100 \mathrm{keV}$ waveband so that we can find out directly what the main contributors to the background are.

\section{CONCLUDING REMARKS}

I am delighted to be able to conclude that real progress has been made in the study of the extragalactic background radiation. I would again caution against expecting to learn too much from diffuse background radiation unless there are very clear spectral signatures. I reaffirm my view that studies of the diffuse background due to discrete sources should be regarded as a means of setting limits to acceptable cosmological models and processes of astrophysical evolution. The astrophysics in the end will have to come from the study of the discrete objects themselves. Until that time, however, the integrated emission from the population of extragalactic objects as a whole will continue to provide important constraints.

The way forward lies largely in the hands of the experimenters and we heard of the many exciting projects which will, we hope, be carried out through the next decade. The list is well known and extensive - ISO, COBE, AELITA, RELIKT-2, IRTS, HUBE, FAUST, FUVIS, ROSAT and so on all have key contributions to make to these studies. If any of the above remarks help the scientists involved in these studies to further their scientific cases, I will feel that this summary has fulfilled a useful purpose.

\section{REFERENCES}

Barcons, X., and Fabian, A. C. 1989, Mon. Not. R. astr. Soc., 237, 119.

Dunlop, J., and Peacock, J. A. 1989, Mon. Not. R. astr. Soc., in press.

Griffiths, R. E. 1989, in Proc. IAU 139, The Galactic and Extragalactic Background Radiation, ed. S. Bowyer and Ch. Leinert, Dordrecht: Kluwer Academic Publishers.

Harrison, E.R. 1987, Darkness at Night: a Riddle of the Universe, Harvard University Press.

Harwit, M. 1989, in Proc. IAU 139, The Galactic and Extragalactic Background Radiation, ed. S. Bowyer and Ch. Leinert, Dordrecht: Kluwer Academic Publishers.

Jones, B. J. T., and Wyse, R. F. G. 1985, Astron. Astrophys., 149, 144.

Longair, M. S. 1984, Theoretical Concepts in Physics, Cambridge University Press.

Longair, M.S. 1989, Alice and the Space Telescope, Johns Hopkins University Press.

Longair, M. S., and Sunyaev, R. A. 1971, Uspekhi Fiz. Nauk, 105, 41.

Martin, C., and Bowyer, S. 1989, Astrophys. J., 338, 677.

Matsumoto, T., Akiba, M., and Murakami, H. 1988, Astrophys. J., 332, 575.

Matsumoto, T., Hayakawa, S., Matsuo, H., Murakami, H., Sato, S., Lange, A.E., and Richards, P.L. 1988, Astrophys. J., 329, 567.

Paresce, F., and Jakobsen, P. 1980, Nature, 288, 119.

Partridge, R. B., and Peebles, P. J. E. 1967, Astrophys. J., 148, 377.

Persic, M., de Zotti, G., Boldt, E.A., Marshall, F.E., Danese, L., Franceschini, A., and Palumbo, G. G. C. 1989, Astrophys. J., 336, L47.

Scheuer, P. A. G. 1974, Mon. Not. R. astr. Soc., 166, 329.

Shectman, S. A. 1974, Astrophys. J., 188, 233. 